\title{
A proof of the minimum principle using flows
}

\author{
by ROBert J. ElliotT* (Edmonton, Alberta, Canada), \\ Michael Kohlmann (Konstanz, F.R.G.) and \\ JACK W. MACKI (Edmonton, Alberta, Canada)
}

Zdzislaw Opial in memoriam

1. Introduction. The theory of stochastic flows was developed by Kunita [4] and Bismut [2]. The concepts and techniques from this theory have been used to discuss the Malliavin calculus [1], and have again returned to deterministic flows, [3]. In this paper, we show how concepts from the theory of deterministic flows can be used to provide an elegant proof of the Pontryagin minimum principle.

2. The dynamics of the optimal control problem as a flow. Consider the control problem

(1) $\dot{x}=f(t, x, u), 0 \leqslant t \leqslant T, x(t) \in \boldsymbol{R}^{d}, x(0)=x_{0}$, with $u(\cdot)$ measurable, $u(t) \in U \subset \boldsymbol{R}^{m}$.

We assume that $f$ is sufficiently well-behaved that:

(a) solutions to initial value problems are unique in $[0, T] \times \boldsymbol{R}^{d}$ and each solution extends to $[0, T]$;

(b) the solution to the $\operatorname{IVP}(1), x(s)=x_{0}$, is a continuously differentiable function of $x_{0}$.

Let the associated cost functional, to be minimized, be defined by:

(2) $\quad c[x(T)], c[\cdot]: \boldsymbol{R}^{d} \rightarrow \boldsymbol{R}$ differentiable.

We remark that (a), (b) need only hold in a "tube" of an appropriate sort about an optimal solution $\left(u^{*}(\cdot), x^{*}(\cdot)\right)$ of $(1),(2)$.

* Member, Applied Mathematics Institute, University of Alberta.

Research of the first and third authors was supported in part by the Natural Sciences and Engineering Research Council of Canada under grants A-7964 (Elliott) and A-3053 (Macki). The research of Elliott was also supported in part by the Air Force Office of Scientific Research, U.S. Air Forces, under grant AF0SR-86-0332. 
The Pontryagin principle states that if $\left(u^{*}(\cdot), x^{*}(\cdot)\right)$ is an optimal solution of (1) (2), then there exists an absolutely continuous $p(\cdot):[0, T] \rightarrow \boldsymbol{R}^{d}$ such that

$$
\min _{v \in U} p(t) \cdot f\left(t, x^{*}(t), v\right)=p(t) \cdot f\left(t, x^{*}(t), u^{*}(t)\right) \text { in }[0, T] \text {. }
$$

In fact $p(\cdot)$ is a solution of the adjoint equation to the linearization of (1) about $x^{*}(\cdot)$.

We will show how this principle follows naturally from the use of ideas from the theory of deterministic flows.

If an initial instant $s \in[0, T]$, an initial value $x \in \boldsymbol{R}^{n}$ and a control $u(\cdot)$ are given, we write the solution of (1) satisfying $x(s)=x$ as $\xi_{s, t}^{u}(x)$. Here the superscript $u$ indicates the dependence on the choice of control $u(\cdot)$; in addition we write $u_{t}$ for $u(t)$. If $\left(u^{*}(\cdot), x^{*}(\cdot)\right)$ is optimal, we write this pair as $\left(u_{t}^{*}, \xi_{0, t}^{*}\left(x_{0}\right)\right)$ and

$$
\xi_{0, t}^{*}\left(x_{0}\right)=x_{0}+\int_{0}^{t} f\left(r, \xi_{0, r}^{*}\left(x_{0}\right), u_{r}^{*}\right) d r .
$$

For any $x \in \boldsymbol{R}^{d}$ and any $s \in[0, T]$ we now define $\xi_{r, s}^{*}(x)$ by the integral equation:

$$
\xi_{s, t}^{*}(x)=x+\int_{s}^{t} f\left(r, \xi_{s, r}^{*}(x), u_{r}^{*}\right) d r .
$$

Notice that $\xi_{s, t}^{*}(x)$ solves (1) as a function of $t$, and takes on the value $x$ at $t=s$, but it is not necessarily optimal (unless $s=0, x=x_{0}$ ). Our assumptions imply that $\xi_{s, t}^{*}(x)$ is a continuously differentiable function of $x$. Differentiating (4) with respect to $x$, we obtain the integral equation defining the matrix $D_{s, t}(x)=\frac{\partial}{\partial x}\left(\xi_{s, t}^{*}(x)\right)$ :

$$
D_{s, t}(x)=I+\int_{s}^{t} f_{x}\left(r, \xi_{s, r}^{*}(x), u_{r}^{*}\right) D_{s, r}(x) d r .
$$

We now define $V_{s, t}(x)$ for any $x \in \boldsymbol{R}^{d}$ and any $s, t$ in $[0, T]$ by the linear integral equation:

$$
V_{s, t}(x)=I-\int_{s}^{t} V_{s, r}(x) f_{x}\left(r, \xi_{s, r}^{*}(x), u_{r}^{*}\right) d r .
$$

LeMma 1 (from [3]). $V_{s, t}(x) D_{s, t}(x)=I$ for all $s, t$ in $[0, T]$, and all $x \in \boldsymbol{R}^{d}$.

Proof. Using (5) and (6), we see that

$$
\begin{aligned}
& V_{s, s}(x) D_{s, s}(x)=I \text {, } \\
& \frac{d}{d t}\left(V_{s, t}(x) D_{s, t}(x)\right)=\left(\frac{\partial V}{\partial t}\right) D+V\left(\frac{\partial D}{\partial t}\right) \\
& =-V_{s, t}(x) f_{x}\left(t, \xi_{s, t}^{*}(x), u_{t}^{*}\right) D_{s, t}(x) \\
& +V_{s, t}(x) f_{x}\left(t, \xi_{s, t}^{*}(x), u_{t}^{*}\right) D_{s, t}(x)=0 .
\end{aligned}
$$


Thus, $V_{s, t}(x)=\left[D_{s, t}(x)\right]^{-1}$ for all $s, t$ in $[0, T]$ and all $x \in R^{d}$. In particular, we conclude that $D_{s, t}(x)$ is always invertible.

Next, for any continuous map (path) $z_{i}:[0, T] \rightarrow \boldsymbol{R}^{d}$, we consider the composite map $\varphi: t \mapsto \xi_{s, t}^{*}\left(z_{t}\right)$, i.e., the function $\varphi(t) \equiv \xi_{s, t}^{*}\left(z_{t}\right)$ defined for $s$ and $t$ in $[0, T]$ by the integral equation:

$$
\varphi(t)=z_{s}+\int_{s}^{t} f\left(r, \varphi(r), u^{*}(r)\right) d r+\int_{s}^{t} D_{s, r}\left(d z_{r} / d r\right) d r .
$$

This equation is obtained from (4) by differentiation with respect to $t$.

We now perturb the given optimal control $u^{*}(\cdot)$ in the by now standard manner:

$$
u_{t}= \begin{cases}u_{t}^{*} & \text { outside }[s, s+h], \\ \tilde{u} \in V & \text { inside }[s, s+h],\end{cases}
$$

and define the curve $z_{t}:[0, T] \rightarrow \boldsymbol{R}^{d}$ by the somewhat improbable integral equation:

$$
z_{t}=x+\int_{s}^{t}\left[D_{s, r}\left(z_{r}\right)\right]^{-1}\left[f\left(r, \xi_{s, r}^{*}\left(z_{r}\right), u_{r}\right)-f\left(r, \xi_{s, r}^{*}\left(z_{r}\right), u_{r}^{*}\right)\right] d r
$$

LEMMA 2.

$$
\xi_{s, t}^{*}\left(z_{t}\right)=\xi_{s, t}^{u}(x) \quad \text { for all } x \in \boldsymbol{R}^{d}, s \text { and } t \text { in }[0, T] .
$$

Proof. By (7) and (9),

$$
\begin{aligned}
\xi_{s, r}^{*}\left(z_{\imath}\right)= & x+\int_{s}^{t} f\left(r, \xi_{s, r}^{*}\left(z_{r}\right), u_{r}^{*}\right) d r \\
& \quad+\int_{s}^{t} D_{s, r}\left(z_{r}\right)\left[D_{s, r}\left(z_{r}\right)\right]^{-1}\left[f\left(r, \xi_{s, r}^{*}\left(z_{r}\right), u_{r}\right)-f\left(r, \xi_{s, r}^{*}\left(z_{r}\right), u_{r}^{*}\right)\right] d r \\
= & x+\int_{s}^{t} f\left(r, \xi_{s, r}^{*}\left(z_{r}\right), u_{r}\right) d r .
\end{aligned}
$$

The assertion follows from the uniqueness of solutions to (1).

3. The minimum principle. If we define $x=\xi_{0, s}^{*}\left(x_{0}\right)$, for a given $s \in[0, T]$, then the optimal cost can be written

$$
c\left[x_{T}^{*}\right]=c\left[\xi_{0, T}^{*}\left(x_{0}\right)\right]=c\left[\xi_{s+h, T}^{*}\left(\xi_{s, s+h}^{*}(x)\right)\right],
$$

for any $h \geqslant 0, s \in[0, T], s+h \in[0, T]$, with $x=\xi_{0, s}^{*}\left(x_{0}\right)$.

Since $u(\cdot)$ cannot give a lower cost than $u^{*}(\cdot)$, thus

$$
c\left[\xi_{s+h, T}^{*}\left(\xi_{s, s+h}^{*}(x)\right)\right] \leqslant c\left[\xi_{s+h, T}^{*}\left(\xi_{s, s+h}^{*}(x)\right)\right]=c\left[\xi_{s+h, T}^{*}\left(\xi_{s, s+h}^{*}\left(z_{s+h}\right)\right)\right],
$$

$$
c\left[\xi_{s, T}^{*}(x)\right]-c\left[\xi_{s, r}^{*}\left(z_{s+h}\right)\right] \leqslant 0
$$


for all $s \in[0, T], h \geqslant 0,(s+h) \in[0, T]$.

This, with judicious application of Mean-Value Theorems and letting $h$ tend to zero, leads to the Pontryagin principle. In fact, by the Mean-Value Theorem,

$$
\left[\frac{\partial c}{\partial \xi}\right] \cdot\left[\left(\xi_{s, T}^{*}(x)\right)-\xi_{s, T}^{*}\left(z_{s+h}\right)\right] \leqslant 0
$$

for $s, h$ as above, where the gradient of $c(\cdot)$ is evaluated somewhere on the line between $\xi_{s, T}^{*}(x)$ and $\xi_{s, T}^{*}\left(z_{s+h}\right)$ in $\boldsymbol{R}^{d}$. Since we will shortly let $h$ decrease to zero, this evaluation point will become $\xi_{s, T}^{*}(x)=x_{T}^{*}$.

It follows from (4) and our assumptions on $f$ that

$$
\left[\frac{\partial c}{\partial \xi}\right] D_{s, T}\left[x-z_{s+h}\right] \leqslant 0
$$

but here the rows of $D_{s . T}=\partial \xi_{s . T}(x) / \partial x$ are evaluated at perhaps different points between $x$ and $z_{s+h}$, because the Mean-Value Theorem is only valid for real-valued mappings and hence must be applied to each component of $\xi_{s, r}^{*}$. From (9) we can write

$$
x-z_{s+h}=\int_{s}^{s+h}\left[D_{s, r}\left(z_{r}\right)\right]^{-1}\left[f\left(r, \xi_{s, r}^{*}\left(z_{r}\right), u_{r}^{*}\right)-f\left(r, \xi_{s, r}^{*}\left(z_{r}\right), u_{r}\right)\right] d r .
$$

Combining (11) and (12), dividing by $h>0$ and letting $h$ go to zero, we obtain (noting that $D_{s s}=I, z_{s}=x=\xi_{s, s}^{*}$ ):

$$
0 \geqslant\left[\frac{\partial c}{\partial \xi}\left(x_{T}^{*}\right)\right] D_{s, T}\left(\xi_{s, s}^{*}\right) \cdot\left[f\left(s, \xi_{s, s}^{*}, u_{s}^{*}\right)-f\left(s, \xi_{s, s}^{*}, \tilde{u}\right)\right]
$$

This is the Pontryagin principle (3) with

$$
p(s)=\left[\frac{\partial c}{\partial \xi}\left(x_{T}^{*}\right)\right] \frac{\partial \xi_{s, T}^{*}}{\partial x}(x)
$$

4. The adjoint equation. By the semigroup property of the solution flows, for $0 \leqslant s \leqslant t \leqslant T$;

$$
\xi_{0, t}^{*}\left(x_{0}\right)=\xi_{s, t}^{*}\left(\xi_{0, s}^{*}\left(x_{0}\right)\right)
$$

Writing $D_{s, t}^{*}=\frac{\partial \xi_{s, t}^{*}}{\partial x}(x)$ and differentiating (14) by the chain rule

$$
D_{0, t}^{*}=D_{s, t}^{*}\left(\xi_{0, s}^{*}\left(x_{0}\right)\right) D_{0 . s}^{*}\left(x_{0}\right)
$$

From (13)

$$
p(s)=\frac{\partial c}{\partial \xi}\left(x_{T}^{*}\right) D_{s, T}^{*}\left(\xi_{s, s}^{*}\right)
$$


Therefore, using (15)

$$
p(s) D_{0, s}^{*}\left(x_{0}\right)=\frac{\partial c}{\partial \xi}\left(x_{T}^{*}\right) D_{0, T}^{*}\left(x_{0}\right)=\text { const. }
$$

Differentiating (16) in $s$

$$
p d D^{*}+(d p) D^{*}=0 .
$$

That is $d p=\left(-p d D^{*}\right) D^{*-1}$. (We have noted in Lemma 1 that $D_{0, s}^{*-1}=V_{0, s}^{*}$ exists.)

From (5)

$$
d D_{0 . s}^{*}=f_{x}\left(s, \xi_{0, s}^{*}\left(x_{0}\right), u_{s}^{*}\right) D_{0, s}^{*} d s .
$$

Therefore, $p(s)$ is the solution of the equation

$$
d p(s)=-p(s) f_{x}\left(s, \xi_{0, s}^{*}\left(x_{0}\right), u_{s}^{*}\right) d s
$$

with initial condition

$$
p(0)=\frac{\partial c}{\partial \xi}\left(x_{T}^{*}\right) D_{0, T}^{*}
$$

\section{References}

[1] J. Baras, R. J. Elliott and M. Kohlmann, The partially observed stochastic minimum principle, preprint, Dept. of Statistics and Applied Probability, University of Alberta, 1987.

[2] J. M. Bismut, Mecanique Aléatoire, Lecture Notes in Math. 886, Springer-Verlag, 1981.

[3] R. J. Elliott, Ordinary differential equations and flows, preprint, Dept. of Statistics and Applied Probability, University of Alberta, 1988.

[4] H. Kunita, Stochastic Partial Differential Equations Connected with Nonlinear Filtering, Lecture Notes in Math. 972, Springer-Verlag, 1983.

DEPARTMENT OF STATISTICS AND APPLIED PROBABILITY

UNIVERSITY OF ALBERTA

EDMONTON, ALBERTA, CANADA

FAKULTÄT FÜR WIRTSCHAFTSWISSENSCHAFTEN UND STATISTIK

UNIVERSITÄT KONSTANZ

KONSTANZ, FEDERAL REPUBLIC OF GERMANY

DEPARTMENT OF MATHEMATICS, UNIVERSITY OF ALBERTA

EDMONTON, ALBERTA, CANADA

Reçu par la Rédaction le 15.06.1988

10 - Annales Polonici Mathernatici LJ 